\title{
Philosophiques
}

\section{La dialectique de l'individu et de la collectivité dans la pensée de Marx}

\author{
Remarques pour une esquisse d'une théorie marxiste des \\ fondements des droits et des libertés humaines
}

\section{André Vachet}

Volume 2, numéro 1, avril 1975

URI : https://id.erudit.org/iderudit/203021ar

DOI : https://doi.org/10.7202/203021ar

Aller au sommaire du numéro

Éditeur(s)

Société de philosophie du Québec

ISSN

0316-2923 (imprimé)

1492-1391 (numérique)

Découvrir la revue

Citer cet article

Vachet, A. (1975). La dialectique de l'individu et de la collectivité dans la pensée de Marx : remarques pour une esquisse d'une théorie marxiste des fondements des droits et des libertés humaines. Philosophiques, 2(1), 23-53.

https://doi.org/10.7202/203021ar d'utilisation que vous pouvez consulter en ligne. 


\section{LA DIALECTIQUE DE L'INDIVIDU ET de LA Collectivité dans LA penSÉe DE MARX}

Remarques pour une esquisse d'une théorie marxiste des fondements des droits et des libertés humaines par André Vachet

Cet exposé sera essentiellement théorique car il ne se propose pas d'évaluer la qualité morale des rapports sociaux dans les sociétés communistes, mais de dégager et de clarifier un certain nombre de notions ou de concepts implicites à la pensée marxiste. Il ne s'agit donc aucunement de faire une critique de la pratique des droits et des libertés humaines dans les sociétés qui se réclament du marxisme, mais de définir les principes de cette critique qui demeure une tâche à accomplir.

Pour clarifier ces principes, je m'excuse de devoir rappeler les principaux éléments de la conception marxiste de l'homme, et plus particulièrement des rapports de l'homme, de l'individu avec la nature et la société. Je devrai le faire en citant les textes mêmes de Marx qui sont souvent ignorés au profit d'une glose réductrice. Cette conception de l'homme détermine le statut de la liberté et aussi celui de la critique marxiste des droits et libertés démocratiques proposés par le libéralisme. Il en résulte l'impossibilité théorique de la dichotomie des droits individuels et des droits collectifs de l'homme, de la liberté individuelle (liberty from) et de la liberté collective (liberty to) ${ }^{1}$. Mais, positivement, cette critique établit un nouveau fondement de la liberté et des droits d'où il est possible d'affirmer de nouvelles formes de la

1. Il faut ici signaler l'approximation toute relative de cette terminologie appliquée au marxisme. Le transfert des termes d'une idéologie dans une autre ne va pas sans ambiguité, confusion et souvent méprise. Dans tous les cas, il s'agit d'un pis-aller. 
liberté et des droits. Par exemple, le droit à la différence, le droit à l'autodétermination et à l'autogestion, le droit à la spécificité culturelle et nationale, le droit à l'espace urbain, à la ville, à la modernité, etc. Drcits qui, confrontés à la pratique des sociétés communistes, les jugent, tout comme la théorie libérale des droits de l'homme, malgré ses limites, a permis et permet encore la critique de la société libérale dite démocratique.

Si nous nous interrogeons sur la représentation spontanée que suscite dans la plupart des cas l'idéal du communisme marxiste, il est plus que probable que nous verrons en premier lieu l'intégration de l'homme dans la société comme suppression de l'individu en tant qu'être autonome. C'est comme si l'idéal marxiste était le reflet de quelque tourment «nirvanique » tendant à fondre et à supprimer la spécificité et la différence individuelle dans l'universalité sociale considérée comme salvatrice. L'homme ne se sauverait qu'en se perdant comme individu concret et qu'en se confondant dans le tout social pour n'avoir d'autre volonté, d'autre passion et d'autre perfection que celle de la totalité.

Cette image s'est profondément enracinée dans la représentation collective d'autant plus qu'il y a indéniablement dans tout individu un désir secret (Freud en démontre les mécanismes) de retourner à l'indifférence originelle en supprimant l'individualité et le poids d'une antonomie ou d'une liberté dont on n'a souvent que faire. Cette image n'est probablement pas étrangère au développement historique du pouvoir dans la société communiste et à la subordination des besoins et du développement individuels aux besoins $t$ t au développement collectifs, subordination allant jusqu'à la suppression morale et même physique des individus. C'est ainsi que certains analystes ont cru que les purges staliniennes et le stalinisme en général étaient constitutifs du système communiste ${ }^{2}$.

Cette destinée du marxisme (et du socialisme en général) est étrange car la moindre attention donnée aux conditions de sa naissance et à ses finalités révèle qu'il fut suscité par la protestation contre la misère de l'individu soumis à un type d'organisa-

2. BUBER-NEUMANN, M., La révolution mondiale, Paris, Casterman, 1971, p. 371. 
tion sociale qui ne pouvait en permettre la réalisation et l'accomplissement comme être rationnel, autonome et libre. Le marxisme et la plupart des socialismes (il y a quelques exceptions partielles - Saint-Simon, etc. - dont l'inspiration déterminante est l'efficacité et la rationalité économique) sont la réaction spontanée et le cri de détresse de l'individualité menacée par la collectivité, elle-même tronquée. On peut même croire que le marxisme tant théorique que pratique est impossible à comprendre si l'on fait abstraction de ce point de départ qui en fixe les finalités ${ }^{3}$.

Nous reviendrons en conclusion sur les raisons de cette destinée pour tenter de montrer qu'au delà de la mauvaise foi de la propagande faisant flèche de tout bois pour discréditer l'adversaire, la croyance dans la suppression de l'individu dans le socialisme marxiste a un fondement réel dans certains éléments structuraux de la pratique historique des sociétés communistes, éléments structuraux d'aspect théorique qui viennent en opposition avec l'ensemble des fondements de la doctrine.

Mais pour l'instant il faut porter l'attention sur ces fondements et tenter de circonscrire la conception marxiste de l'homme ${ }^{4}$. La lecture de l'oeuvre même de Marx est ici essentielle.

3. Voir entre plusieurs l'analyste chrétien Pierre Bigo, Marxisme et bumanisme, introduction à l'oeuvre économique de Karl Marx, Paris, P.U.F., 1961, p. 132 et suivantes.

4. Depuis environ dix ans, non seulernent les marxistes occidentaux, spécialement les Italiens recourant à l'inspiration de Gramsci, mais surtout les penseurs de l'Europe de l'Est ont profondément analysé la conception marxiste de l'homme (réflexion qu'on peut soupçonner de ne pas être étrangère aux tentatives de libéralisation en Tchécoslovaquie et en Pologne). Parmi les cuvres traduites les plus importantes, il faut citer :

GOULIANE, C.I., Le marxisme devant l'bomme, essai d'antbropologie pbilosopbique, Paris, Payot, 1968 (Roumanie);

KOSIK, K., La dialectique du concret, Paris, Maspéro, 1970 ('Tchécoslovaquie) ;

PETROVIC, G., Marx in the Mid-Twentieth Century, Anchor Books, Doubleday, N.Y. 1967 (voir spécialement « Marx's concept of man »; « Man as economic animal and man as praxis»; "What is freedom ?») (Yougoslavie) ;

SCHAFF, A., Le marxisme et l'individu, contribution à la pbilosophie marxiste de l'bomme, Paris, A. Colin, 1968 (Pologne) ;

KOLAKOWSKI, D., Toward a Marxist Humanism, Essays on the Left Today, N.Y. Grove Press, 1968 (Pologne).

On peut rapidement prendre conscience du sens de ces recherches dans la plupart des textes qu'Eric Fromm a réunis dans Socialist Humanism, an International Symposium, Doubleday, N.Y., 1965. 
Cette lecture est éclairante car elle révèle de façon incontestable et en deçà de toute interprétation que le point de départ et le fondement de la pensée marxiste est l'homme, l'homme concret, l'individu concret. Sans accumuler les citations, ce serait à la fois trop facile et fastidieux, il faut tout de même rappeler quelques textes.

Les présuppositions par quoi nous commençons ne sont pas des présuppositions arbitraires, ce ne sont pas des dogmes, ce sont des présuppositions téelles dont on ne peut faire abstraction que dans l'imagination. Ce sont les individus réels, leur action et leurs conditions d'existence matérielles, soit déjà existantes, soit produites par leur propre action. Ces présuppositions sont donc constatabules par la voie purement empirique. La première présupposition de toute histoire des hommes, c'est naturellement l'existence d'individus humains vivants. Le premier état de choses à constater, c'est donc l'organisation corporelle de ces individus et le rapport où cela les met avec le reste de la nature ${ }^{5}$.

Ou encore de façon plus explicite:

Une révolution sociale... se trouve placée au point de vue de l'ensemble, parce qu'elle constitue une protestation de l'homme contre la vie, indigne d'un être humain, qu'on lui impose, parce qu'elle part du point de vue de chaque individu réel, parce que la collectivité dont il s'efforce de ne plus être isolé est la collectivité véritable des hommes, l'essence humaine ${ }^{6}$.

Et il ne s'agit pas seulement d'une position des oeuvres de jeunesse de Marx, car il écrit dans l'Introduction à la critique de l'économie politique en 1857 :

Des individus qui produisent en société - donc une production d'individus socialement déterminée - tel est naturellement le point de départ ${ }^{7}$.

Le principe même de la pensée de Marx apparâit donc être l'homme concret, l'homme empirique doué d'une nature spéci-

5. MARX, K., Idéologie allemande, Oeuvres pbilosopbiques, Paris, A. Costes, 1953, vol. VI p. 154.

6. MARX, K., Le roi de Prusse et la réforme sociale, Oeuvres pbilosopbiques, Paris, A. Costes, vol. V, p. 243.

7. MARX, K., Contribution à la critique de l'économie politique, Paris, A. Costes, 1954, p. 263. 
fique et inscrit dans une histoire avec ses passions, ses désirs, ses besoins, sa misère. C'est d'ailleurs pourquoi on trouverait difficilement une définition de l'homme chez Marx. Ce point de départ qui résiste à l'universel et à l'universalisable est la raison du rejet à la fois de la conception matérialiste traditionnelle et de la conception idéaliste de l'homme. Rejet que Marx fonde en dénonçant l'homme abstrait et universel de Hegel, l'homme dont la réalité est le Soi qui ne tient positivement son essence que par l' «Esprit Absolu».

Le Soi n'est que l'homme saisi abstraitement et engendré par l'abstraction (...) Quand l'homme réel, en chair et en os, campé sur la terre solide et bien ronde, l'homme qui aspire et expire toutes les forces de la nature, pose ses forces essentielles objectives réelles par son aliénation comme des objets étrangers, ce n'est pas le fait de poser qui est sujet; c'est la subjectivité de forces essentielles objectives, dont l'action doit être également objective (...) Nous voyons ici que le naturalisme conséquent, ou humanisme, se distingue aussi bien de l'idéalisme que du matérialisme et qu'il est en même temps leur vérité qui les unit (...) L'homme est immédiatement être de la nature. En qualité d'être naturel, et d'être naturel vivant, il est d'une part pourvu de forces naturelles, de forces vitales; il est un être naturel actif; ces forces existent en lui sous la forme de dispositions et de capacités, sous forme d'inclinations (...) Dire que l'hormme est un être en chair et en os, doué de forces naturelles, vivant, réel, sensible, objectif, c'est dire qu'il a pour objet de son être, de la manifestation de sa vie, des objets réels, sensibles, et qu'il ne peut manifester sa vie qu'à l'aide d'objets réels, sensibles. Être objectif, naturel, sensible, c'est la même chose qu'avoir en dehors de soi objet, nature, sens ou qu'être soi-même objet, nature, sens pour un tiers ${ }^{8}$.

C'est aussi parce que Marx part de l'être naturel concret, de l'individu concret que celui-ci est toujours inscrit dans un rapport social comme le conclut le texte qui précède. L'homme est toujours imbriqué dans un ensemble social, est toujours un être social, non pas nécessairement comme entité ontologique, là n'est pas le point de vue de Marx, mais comme homme vivant,

8. MARX, K., Manuscrits de 1844, Économie politique et philosopbie, Paris, Éditions sociales, 1962, pp. 134 et 136. 
comme «être en chair et en os», comme être particularisé, historique.

Les individus sont toujours partis d'eux-mêmes, mais naturellement d'eux-mêmes dans le cadre de leurs conditions et de leur situation historique données et non pas de «l'individu pur» au sens des idéologues ${ }^{9}$.

Et Marx de railler les «robinsonades » des libéralistes du XVIIIe siècle dissertant sur l' «état de nature ${ }^{10}$. La condition existentielle de l'homme est toujours sa condition sociale.

L'homme concret se situe donc pour Marx et le marxisme à l'articulation de la nature et de la société. C'est là précisément la source de plus d'une interprétation faussée, car l'individu et la société sont «de» la nature. L'homme n'est pas social en deçà ou au-delà de la nature et de sa nature, mais par sa condition d'être naturel. Et la société n'est pas indépendante de l'homme, de l'individu, car elle en dépend et il en est le contenu. C'est le sens que Marx accorde à l'affirmation suivante:

L'homme n'est pas un être abstrait, extérieur au monde réel. L'homme c'est le monde de l'homme, l'État, la société ${ }^{11}$.

La société est d'abord intrinsèque à l'homme avant d'être son milieu. Il y a coïncidence de l'individualité et de la sociabilité de l'homme concret: toute tentative pour opposer ces deux dimensions conduit à les nier l'une et l'autre, c'est-à-dire à tronquer l'individu tout autant que la société, comme Marx l'implique quand il écrit :

Il faut surtout éviter de fixer de nouveau la «société 》 comme une abstraction en face de l'individu. L'individu est l'être social. La manifestation de sa vie - même si elle n'apparaît pas sous la forme immédiate d'une manifestation collective de la vie, accomplie avec d'autres et en même temps qu'eux - est donc une manifestation et une affirmation de la vie sociale. $\mathrm{La}$ vie individuelle et la vie générique de l'homme ne

9. MARX, K., Idéologie allemande, A. Costes, vol. VI, p. 226.

10. MARX, K., Contribution à la critique de l'économie politique, A. Costes, pp. $149-150$.

11. MARX, K., Contribution à la critique de la pbilosopbie du droit, A. Costes, Oeuvres pbilosopbiques, vol. I, p. 84 . 
sont pas différentes, malgré que - et ceci nécessairement - le mode d'existence de la vie individuelle soit un mode plus particulier ou plus général de la vie générique ou que la vie du genre soit une vie individuelle plus particulière ou plus générale (...) L'homme - à quelque degré qu'il soit donc un individu particulier (et sa particularité en fait précisément un individu et un être social individuel réel) - est donc tout autant la totalité, la totalité idéale, l'existence subjective pour soi de la société pensée et sentie, que, dans la réalité, il existe soit comme contemplation et jouissance réelle de l'existence sociale soit comme totalité de manifestation humaine de la vie ${ }^{12}$.

Concrètement, l'individu intègre la société à lui-même tout autant qu'il s'intègre à la société. Le marxisme continue et dépasse la vieille tradition aristotélicienne. L'extériorisation de la société et sa réduction à un pur moyen vient de la rupture de cette tradition avec le triomphe de l'idéologie libérale au XVIIIe siècle. Elle est d'ailleurs de nature idéologique.

Plus on remonte dans le cours de l'histoire, plus lindividu (...) apparaît dans un état de dépendance, membre d'un ensemble plus grand: cet état se manifeste tout d'abord de façon tout à fait naturelle dans la famille et dans la famille élargie jusqu'à former la tribu, puis dans les différentes formes de communautés, issues de l'opposition et de la fusion des tribus. Ce n'est qu'au XVIIIe siècle, dans la «société bourgeoise», que les différentes formes de l'ensemble social se présentent à l'individu comme un simple moyen de réaliser ses buts particuliers, comme une nécessité extérieure. Mais l'époque qui engendre ce point de vue, celui de l'individu isolé, est précisément celle où les rapports sociaux (revêtant de ce point de vue un caractère général) ont atteint le plus grand développement qu'ils aient connu. L'homme est, au sens le plus littéral, un animal politique, non seulement un animal social, mais un animal qui ne peut s'isoler que dans la société ${ }^{13}$.

12. MARX, K., Manuscrits de 1844, op. cit. p. 90.

13. MARX, K., Contribution à la critique de l'économie politique, op. cit. pp. 149-150. L'édition des cuvres complètes de Marx emploie «qui ne peut s'individualiser que dans la société » au lieu de «s'isoler», p. 237. M. Rubel emploie plutôt «singulariser». Pages choisies pour une étbique socialiste, M. Rivière, Paris, 1948, p. 64. 
C'est dans ce sens précis que Marx, refusant l'abstraction feuerbachienne qui considérait l'être humain uniquement en tant que "genre», en tant qu'universalité interne, muette, liant d'une façon purement naturelle les nombreux individus, affirme que "l'essence humaine n'est pas une abstraction inhérente à l'individu isolé. Dans sa réalité, elle est l'ensemble des rapports sociaux $\gg^{14}$.

Mais si le rapport individu-société est réel, naturel, au point de constituer une réalité indivisible en tant que telle, il faut se garder de toute interprétation qui réduirait un terme à l'autre, et, pour s'opposer au libéralisme qui mine l'essentialité de la société, supprimerait l'individualité dans la société. Le rapport individu-société est l'essentiel qui ne permet pas l'élimination de l'un des éléments. Il se constitue comme mouvement circulaire ou comme spirale et chacun des éléments en est un des moments essentiels, comme l'explicite la troisième Thèse sur Feuerbach:

La doctrine matérialiste qui veut que les hommes soient des produits des circonstances et de l'éducation, que, par conséquent, des hommes transformés soient des produits d'autres circonstances et d'une éducation modifiée, oublie que ce sont précisément les hommes qui transforment les circonstances et que l'éducateur a lui-même besoin d'être éduqué ${ }^{15}$.

C'est dans cette perspective qu'il faut comprendre des affirmations comme la suivante:

Ce n'est pas la conscience qui détermine la vie, c'est la vie qui détermine la conscience ${ }^{16}$.

Ou, comme le fameux énoncé, presque identique, de la Préface de la Contribution à la critique de l'économie politique, qui a été si mal compris par toutes les interprétations vulgaires du marxisme qui l'ont réduit, à la fois, à un matérialisme brut et à un pur collectivisme :

Le mode de production de la vie matérielle conditionne le processus de vie social, politique et intellectuel en général.

14. MARX, K., Thèses sur Feuerbach, VI, Études philosophiques, Paris, Éditions sociales, 1961, p. 63.

15. Ibidem, p. 62.

16. MARX, K., Idéologie allemande, A. Costes, vol. VI, p. 158. 
Ce n'est pas la conscience des hommes qui détermine leur être; c'est inversement leur être social qui détermine leur conscience ${ }^{17}$.

Il est entendu que l'état de la société et le développement historique de la conscience sociale conditionnent l'individu dans une société donnée. Donc, en un certain sens, les rapports sociaux créent la conscience et l'individu concret - sans quoi il faudrait se reporter à une forme d' « innéisme». Mais non seulement il ne faut pas y voir un déterminisme unilatéral, il faut au contraire y reconnaître la priorité de l'action et de l'affirmation de l'homme concret qui jouit d'un pouvoir d'initiation. Sans quoi, comment la révolution aurait-elle un sens? comment pourraitelle être un événement politique dépendant ultimement de la volonté des hommes? Comment la résistance à l'oppression et à l'exploitation serait-elle possible? Comment la société répressive ne se reproduirait-elle pas indéfiniment? D'ailleurs, c'est le sens même du communisme qui permet d'assurer définitivement la priorité de l'homme et de le libérer des forces aveugles. En effet, comme l'écrit Engels :

La lutte pour l'existence individuelle cesse. Par là, pour la première fois, l'homme se sépare, dans un certain sens, définitivement du règne animal, passe de conditions animales d'existence à des conditions réellement humaines. Le cercle des conditions de vie entourant l'homme, qui jusqu'ici dominair l'homme, passe maintenant sous la domination et le contrôle des hommes, qui pour la première fois, deviennent des maîtres réels et conscients de la nature, parce que et en tant que maîtres de leur vie en société. Les lois de leur propre pratique sociale qui, jusqu'ici, se dressaient devant eux comme des lois naturelles, étrangères et dominatrices, sont dès lors appliquées par les hommes en pleine connaissance de cause, et par là dominées. La vie en société propre aux hommes qui, jusqu'ici, se dressait devant eux comme octroyée par la nature et l'histoire, devient maintenant leur acte propre et libre. Les puissances étrangères, objectives qui, jusqu'ici, dominaient l'histoire, passent sous le contrôle des hommes eux-mêmes. Ce n'est qu'à partir de ce moment que les hommes feront eux-mêmes leur histoire en pleine conscience; ce n'est qu'à partir de ce moment que les

17. Editions sociales, p. 4. 
causes sociales mises par eux en mouvement auront aussi, d'une façon prépondérante et dans une mesure toujours croissante, les effets voulus par eux. C'est le bond de l'humanité, du règne de la nécessité, dans le règne de la liberté ${ }^{18}$.

Marx est tout aussi explicite pour défendre le pouvoir de l'individu — au moins comme possibilité — dans la circularité des conditionnements et des déterminations :

À l'époque actuelle la domination des considérations matérielles sur les individus, l'écrasement de l'individualité par l'accidentel a pris la forme la plus accusée et la plus universelle et fixé par là-même aux individus existants une tâche tout à fait déterminée. Elle leur a fixé la tâche de remplacer la domination des conditions et du hasard sur les individus par la domination des individus sur le hasard et les conditions (...) elle leur a prescrit de se libérer d'un mode tout à fait déterminé de développement. Cette tâche prescrite par les conditions présentes coïncide avec la tâche de donner à la société une organisation communiste ${ }^{19}$.

Et donc, si l'action des hommes est conditionnée, elle n'est pas pré-établie ni fixée de façon univoque, elle demeure créatrice même si elle est produite par la nature, par la société, par l'histoire, comme le suppose la diversité même de l'action des hommes. Si l'on n'admettait pas cette priorité et cette autonomie relative du dynamisme humain, toutes les thèses de Marx sur le travail, la praxis, l'autocréation, crouleraient dans la contradiction et l'absurdité. Le travail par exemple, transforme la réalité objective, c'est-à-dire la nature, la société et par suite l'homme luimême comme être naturel et être social. Il est concrètement

18. ENGELS, F., Anti-Dühring, Paris, Éditions sociales, 1963, p. 322.

19. MARX, K., Idéologie allemande, Oeuvres pbilosopbiques, A. Costes, vol. IX, p. 97. «Les rapports de dépendance personnelle (d'abord tout à fait naturels) sont les premières formes sociales dans lesquelles la productivité humaine se développe lentement et d'abord en des points isolés. L'indépendance personnelle, fondée sur la dépendance à l'égard des cboses est la deuxième grande étape : il s'y constitue pour la première fois un système général de métabolisme social, de rapports universels, de besoins diversifiés et de capacités universelles. $\mathbf{L a}$ troisième étape, c'est la libre individualité fondée sur le développement universel des hommes et sur la maîtrise de leur productivité sociale et collective ainsi que de leurs capacités sociales. La seconde crée les conditions de la troisième »Idem, Fondements de la critique de l'économie politique, Paris, Anthropos, 1968, vol. I, p. 95. 
auto-création de l'homme: quand ce processus d'autocréation cesse, l'homme s'est aliéne et s'est réifié ${ }^{20}$. Le travail crée donc la réalité humaine cornme produit, il transforme la nature et la société en humanité. Par là, il les subordonnerait et les dominerait si elles étaient des réalités distinctes et opposées, si elles n'étaient pas la continuité même de l'homme. C'est d'ailleurs ce pouvoir de s'abstraire de son milieu (biologique et social), mais sans le nier ou l'éliminer, qui fait la spécificité de l'homme:

L'animal s'identifie directement avec son activité vitale. Il ne se distingue pas d'elle. Il est cette activité. L'homme fait de son activité vitale elle-même lobjet de sa volonté et de sa conscience. Il a une activité vitale consciente. Ce n'est pas une détermination avec laquelle il se confond directement. L'activité consciente distingue directement l'homme de l'activité vitale de l'animal ${ }^{21}$.

Ici s'inscrit la conception marxiste de la liberté, mais l'on comprend sans doute mieux celle-ci si l'on résume la description de l'homme en se référant aux concepts de totalité et de totalité partielle ou ouverte.

Pour Marx l'individu est un tout et, comme tel, il est autonomie et pouvoir sans lequel l'autonomie serait illusoire. Il se pose comme totalité et il s'affirme en pliant les circonstances à sa volonté. Mais cet homme, cet individu, n'est pas une magnifique monade close (Leibniz) ni la «solitude ultime et effrayante » de certains scolastiques (Duns Scot). Il est une totalité partielle ouverte à d'autres totalités : la nature, la société, parce que, tout en le dépassant comme unité, elles se retrouvent en lui. C'est ce que C.I. Gouliane décrit bien :

Dans la pensée de Marx l'homme concret est un tout, tant pour ses possibilitési que pour la réalisation de soi. Toutefois, la réalisation de ses virtualités ne dépend pas uniquement de l'individu, elle dépend aussi d'un certain nombre de conditions objectives ${ }^{22}$.

20. Idem, Manuscrits de 1844, Editions Sociales, p. 57 et suivantes.

21. MARX, K., Manuscrits de 1844, Éd. Sociales, p. 63.

22. Le marxisme devant l'bomme, p. 39. Voir aussi A. Schaff, Le marxisme et l'bomme, p. 113. 
La totalité qu'est l'individu n'est donc pas achevée tant que toute la nature et toute la société ne sont pas réalisées en lui-même, tant qu'elles demeurent extériorité et pouvoir sur l'individu, c'est-à-dire altérité ou aliénation. Or, c'est là l'accomplissement de la révolution, le sens de l'appropriation: réduire et supprimer l'altérité de la nature et de la société pour permettre la pleine spontanéité et la pleine liberté de l'homme.

Mais, d'autre part, il est entendu que la totalité «totalisante », celle qui enveloppe et achève les totalités partielles, n'est réductible ni à la société - il s'agirait alors d'une collectivisation des totalités partielles et leur négation: elle serait une totalité abstraite - ni à la nature qui produirait alors un déterminisme absolu détruisant l'autonomie des totalités partielles et de leurs mouvements au profit de la constitution d'une structure qui ne soit que structure. L'achèvement de la totalité ne peut s'accomplir que par l'achèvement des totalités partielles, c'est-àdire par la réalisation positive et le maintien des rapports entre les individus, la société et la nature. C'est seulement dans ce cas que la totalité finale sera réelle, parce que concrète, «le concept (étant) concret, parce qu'il est la synthèse de multiples déterminations, donc unité de la diversité ${ }^{23}$.

Dans cette totalité concrète dont les éléments sont inséparables sans qu'elle cesse d'être, l'homme intègre ses contraires, c'est-à-dire son extériorité à son intériorité, maintenant la tension de l'une par rapport à l'autre ${ }^{24}$; mais l'homme qui reçoit objectivement son extériorité, la crée aussi. C'est pourquoi Lénine pouvait écriše :

La conscience humaine ne reflète pas seulement le monde objectif, elle le crée ${ }^{25}$.

23. MARX, K., Contribution à la critique de l'économie politique, A. Costes, p. 165.

24. « Le concept ( $=$ ho), en tant qu'être subjectif, suppose, à son tour, l'existence d'un autre en soi (la nature indépendante de l'homme). Ce concept (= l'homme) est la tendance à se réaliser soi-même, à se donner par ses propres moyens l'objectivité dans un monde objectif et à se réaliser (à s'accomplir) soi-même ». Lénine, Cabiers pbilosophiques, cité in Gouliane, op. cit., p. 164.

25. Cité, Ibidem, p. 161. 
L'homme est conctètement le moteur et le créateur de sa propre affirmation en s'emparant et en contrôlant les conditions de son existence. Marx écrit bien :

$\grave{A}$ toutes les époques et dans toutes les circonstances, les individus sont «partis d'eux-mêmes 》, mais comme ils r'étaient jamais uniques en ce sens qu'ils n'auront pas eu besoin de rapport entre eux, comme leurs besoins - donc leur nature - et la manière de les satisfaire créaient entre eux des liens récipro. ques $(. .$.$) , ils étaient obligés d'établir des relations entre eux.$ En outre, comme ils entraient en communication non pas comme des moi purs mais en tant qu'individus se trouvant à un degré déterminé de l'évolution de leurs forces productives et de leurs besoins, communication qui, à son tour, déterminait la production et les besoins, c'était précisément le comportement personnel, individuel, des individus, leur comportement réciproque en tant qu'individus, qui créait et crée quotidiennement de nouveau les conditions existantes ${ }^{20}$.

C'est cette prise en charge et cette domination sur les conditions de leur vie matérielle, culturelle, et intellectuelle, qui permet « la transformation progressive des individus en individus totals ${ }^{27}$.

L'individu comme totalité jouit d'un pouvoir «totalisant» dont la fin est l'appropriation de la totalité de la réalité humaine (intériorité-extériorité). «L'homme s'approprie son être universel d'une manière universelle, donc en tant qu'homme total ${ }^{28}$. C'est-à-dire que pour l'homme le monde extérieur n'est pas exclusivement extérieur comme le monde objectif n'est pas exclusivement objectif.

Mais l'appropriation par l'individu des conditions de son existence ne se fait pas dans l'abstraction et dans l'isolement, car elle serait illusoire. C'est par l'intermédiaire de la société, de l'association des individus que ceux-ci soumettent à leur contrôle «les conditions de leur libre développement et de leur libre mouvement, conditions jusque-là livrées au hasard ${ }^{29}$. Il ne

26. Idéologie allemande, in M. Rubel, op. cit., pp. 266-267.

27. Ibidem, p. 333. Voir aussi note 12.

28. MARX, K., Manuscrits de 1844, Éd. Sociales, p. 91.

29. MARX, K., Idéologıe allemande, trad. Rubel, p. 335. 
s'agit aucunement ici de soumettre les individus à la société. Une telle soumission et le contrôle des conditions externes sur l'homme implique pour Marx l'absence du développement humain et des conditions sociales de la vie ${ }^{30}$. Mais même arrivée à son plein développement, la condition concrète de l'homme fait que son affirmation passe par la société (et la nature) qui devient « une association cù le libre épanouissement de chacun est la condition du libre épanouissement de tous ${ }^{31}$. L'intérêt de la société ne se distingue pas de l'intérêt des individus même si cette coïncidence des intérêts n'est pas toujours manifeste dans chacune des situations complexes. C'est pourquoi Marx et Engels peuvent conclure :

Les communistes ne songent guère à abolir «l'individu particulier » au profit de l'ho «général 》 plein de dévouement (...) Les théoriciens communistes se distinguent précisément par le fait d'avoir été les seuls aussi à découvrir la vérité que voici : l'histoire prouve que ce sont toujours des «particuliers》 qui ont créé «l'intérêt général ». Ils savent que cette opposition n'est qu'apparente, parce que le caractère dit « général 》 jaillit toujours de l'intérêt particulier, auquel il ne s'oppose jamais en tant que force indépendante, car il possède une histoire indépendante; aussi cette opposition se trouve-t-elle en réalité constamment abolie pour se reproduire sans cesse ${ }^{32}$.

Si l'analyse qui précède est juste dans l'ensemble, le cadre et le sens de la liberté se trouvent à être définis par la situation de l'homme comme totalité. En effet, la liberté qui peut être attribuée à l'homme est précisément celle qui tient au caractère de totalité spécifique de l'individu, donc à son achèvement, à sa réalisation et à ses possibilités, mais en même temps cette liberté est située dans l'inachèvement même de l'individu qui grève son autonomie et son unicité par rapport aux autres individus, à la société et à la nature, elle est située dans la qualité même du rapport entre l'intériorité et l'extériorité de l'individu et en a les caractéristiques. Totalité partielle et ouverte, l'individu est, à la fois, unique, autonome et conditionné, mais sans que ce condi-

30. MARX, K., Fondements de la critique de l'économie politique, Anthropos, vol. I, pp. 99 et 101 .

31. MARX, K., Manifeste Communiste, Paris, Editions sociales, 1960, p. 35.

32. Cité in Gouliane, op. cit., p. 171. 
tionnement n'épuise les possibilités de l'individu. Le processus d'objectivation qui soumet l'individu est lui-même partiel, justement parce qu'il est rapport à l'individu. La liberté ne peut donc pas être absolue, pas plus que l'individu n'est absolu, mais elle existe réellement (concrètement), au travers des conditionnements, dans la mesure où ceux-ci ne sont pas eux-mêmes absolus, dans la mesure même' où l'homme demeure le moteur et le créateur de son propre développement.

Adam Schaff a bien compris la condition de la liberté pour l'individu quand il écrit :

Il ne s'agit pas en effet de ce que le choix ne soit conditionné par rifn, l'important c'est qu'il soit conscient et non pas imposé par des forces extérieures à l'homme. Le degré de liberté de l'homme ne dépend pas en effet, de la mesure dans laquelle il est renfermé sur lui-même et détaché des conditionnements sociaux (ce qui, d'ailleurs, est une fiction) mais de la mesure dans laquelle il est conscient des objectifs à atteindre et des moyens pour y parvenir, en effectuant un choix parmi les possibilités d'action.

Selon le marxisme, l'action des hommes est toujours socialement conditionnée, mais jamais elle n'est fixée une fois pour toutes de manière univoque. En partant de processus historiques objectifs, le choix de l'action humaine est donc conditionné, mais il r'est pas imposé et demeure fondé sur la réflexion consciente, donc en ce sens libre. C'est précisément pourquoi l'homme n'est pas l'instrument aveugle de forces qui le dominent, mais il est le créateur de l'histoire. Cela signifie non seulement que l'homme crée parce qu'il agit, mais surtout parce qu'il agit délibérément, qu'il effectue un choix conscient entre diverses possibillités ${ }^{33}$.

Le problème de la liberté pose celui de la nécessité. La liberté tient à l'extériorisation qui permet l'expression de la spontanéité jusque dans l'extériorité ou la nécessité. La nécessité tient pour sa part à la limite de l'intériorisation toujours inache-

33. Ibidem, pp. 163 et 164 . La même idée se retrouve chez Gouliane, op. cit., pp. 164, 165, 202 et 208; chez Petrovic, "What is freedom?》, op. cit., p. 115 et suivantes; chez Lucien Goldmann, Marxisme et sciences bumaines, Coll. «Idées », Paris, Gallimard, 1970, pp. 175-178. 
vée pour une totalité ouverte. Marx est encore plus explicite quand il écrit dans Le Capital:

À la vérité, le règne de la liberté commence seulement à partir du moment où cesse le travail dicté par la nécessité et les fins extérieures; il se situe donc par sa nature même au-delà de la sphère de la production matérielle proprement dite (...)

L'empire de la nécessité n'en subsiste pas moins. C'est au-delà que commence l'épanouissement de la puissance humaine qui est sa propre fin, le véritable royaume de la liberté, qui cependant ne peut fleurir qu'en se fondant sur le royaume de la nécessité ${ }^{34}$.

Par rapport à l'extériorité, parce qu'il n'y a pas coupure avec l'intériorité, la liberté existe dans la mesure même de la continuité.

Dans ce domaine (celui du processus productif), écrit encore Marx, la liberté ne peut consister qu'en ceci: les producreurs associés - l'homme socialisé — règlent de manière rationnelle leurs échanges organiques avec la nature et les soumettent à leur contrôle commun, au lieu d'être dominés par la puissance aveugle de des échanges; et ils les accomplissent, en dépensant le moins d'énergie possible, dans les conditions les plus dignes, les plus conformes à leur nature humaine ${ }^{35}$.

C'est pourquoi la liberté a toujours un rapport à la société car en un sens concret, elle "n'est pas possible sans la communauté. Ce n'est que dans la communauté que l'individu acquiert les moyens de développer ses facultés dans tous les sens; ce n'est que dans la communauté que la liberté devient possible ${ }^{36}$.

Mais le problème de la liberté, justement parce que celle-ci est limitée et menacée, parce qu'elle est celle de l'homme concret, n'est pas statique. La liberté, c'est en premier lieu la reproduction de la libération: elle est l'autocréation de l'homme par l'homme, son auto-émancipation qui ne peut que passer par la libération de tout l'homme. La liberté c'est pour l'homme la première finalité

34. Tome II, Oeuvres de Karl Marx, La Pléiade, Paris, Gallimard, 1968, vol. II, pp. 1487-1488.

35. Ibidem, pp. 1487-1488.

36. MARX, K., Idéologie allemande, A. Costes, vol. VI, p. 226. 
et la justification de la révolution qui consiste précisément dans ce «bond de l'humanité, du règne de la nécessité, dans le règne de la liberté ${ }^{37}$.

Dans ce contexte surgit l'opposition entre la liberté individuelle et la liberté collective, mais cette opposition est phénoménale et illusoire car, fondamentalement, la liberté individuelle ne peut être complète sans la liberté collective et la liberté collective ne peut être réelle sans la liberté individuelle. La lutte pour la diberté collective est aussi la lutte pour la liberté des individus, car il ne peut y avoir de société libre sans individus libres ${ }^{38}$.

Là précisément se fondent, comme conséquence de la situation spécifique de l'homme et de son statut d'être libre à l'intérieur de l'ensemble social, la conception marxiste des droits et des libertés et la critique marxiste des «droits de l'homme» défendus par le libéralisme.

En effet, le droit tient à ce que l'homme est sujet, c'est-à-dire une totalité comme le suppose toute la théorie de l'aliénation ${ }^{39}$. En tant que totalité, il est autonome, unique et source de possibilité et de perfection. Le droit s'y fonde mais non pas comme droit absolu vu que la totalité est ouverte et inachevée, que l'autonomie est relacive et en voie d'accomplissement.

Mais le droit ainsi conçu n'est ni un droit individuel, ni un droit collectif au sens où le libéralisme les entend et les oppose. L'opposition des droits individuels et des droits collectifs est absurde dans une doctrine où l'individu est totalité partielle dans une totalité totalisante dont les éléments sont l'individu (les individus), la nature et la société. C'est dire que tout droit individuel est à la fois naturel et social et tout droit social est individuel et naturel. La conception de l'homme à la base du marxisme et la conception de la liberté qui en découle ne peuvent permettre aucune autre interprétation.

Marx est terriblement silencieux sur la question du droit, tout autant que sur le statut des droits et libertés de l'nomme. Mais

37. ENGELS F., Anti-Dubring, Éditions Sociales, p. 322. Marx, Ibidem, vol. IX, p. 97.

38. PETROVIC, op. cit., pp. 128-130.

39. MARX, K., Manuscrits de 1844, Éd. sociales, pp. 68-69; P. Bigo, op. cit., pp. 138-139. 
la critique qu'il fait de la conception libérale de ces droits et libertés est significative, car sa condamnation tient essentiellement à la dénonciation de leur caractère abstrait par suite de leur fondement exclusif dans l'individu isolé et suffisant. Les droits du libéralisme ne visent pas à l'émancipation de l'homme concret parce qu'il ne vise l'homme que dans sa partialité et dans son abstraction. L'émancipation de l'homme ne peut être réelle sans être totale, impliquant la nature en l'homme comme son rapport aux autres.

Ce n'est pas que les droits individuels du libéralisme soient sans signification : ils correspondent à une émancipation politique de l'homme. Ils sont donc un acquis car:

l'émancipation politique constitue, assurément, un grand progrès. Elle n'est pas, il est vrai, la dernière forme de l'émancipation humaine, mais elle est la dernière forme de l'émancipation humaine dans l'ordre du monde actuel. Entendons-nous bien: nous parlons ici de l'émancipation réelle, de l'émancipation pratique ${ }^{40}$.

La théorie des droits, comme celle de la démocratie constitutionnelle ${ }^{41}$ qui s'y rattache, sont les stages les plus avancés de l'idéologie libérale et de l'organisation capitaliste de la société.

Marx rejette les droits du libéralisme parce qu'ils consacrent «la séparation de l'homme, de sa communauté, de luimême et des autres hommes » ${ }^{42}$. Ils reposent sur une fragmentation de la totalité humaine individuelle. Or, la fragmentation de la totalité en l'homme est l'aliénation elle-même. C'est dans ce sens que Marx écrit :

On fait une distinction entre les «droits de l'homme» et les «droits du citoyen». Quel est cet «homme» distinct du citoyen? Personne d'autre que le membre de la société bourgeoise. Pourquoi le membre de la société bourgeoise est-il appelé «homme», homme tout court, et pourquoi ses droits sont-ils appelés droits de l'homme? Qu'est-ce qui explique ce fait?

40. MARX, K., La question juive, Coll. 10-18, Union générale d'éditions, Paris, 1968 , p. 26.

41. Voir l'article intéressant de M. Rubel, «Le concept de démocratie chez Marx », in Contrat social, vol. VI, no 4.

42. MARX, K., La Question juive, op. cit., p. 27. 
Par le rapport de l'État politique à la société bourgeoise, par l'essence de l'émancipation politique. Constatons avant tout le fait que les «droits de l'homme», distincts des «droits du citoyen $»$, ne sont rien d'autre que les droits du membre de la société bourgeoise, c'est-à-dire de l'homme égoïste, de l'homme séparé de l'homme et de la communauté ${ }^{\mathbf{4 3}}$.

Chacun des droits n'est que l'affirmation de l'individu abstrait, de l'individu-monade. Par exemple,

la liberté est le droit de faire tout ce qui ne nuit pas à autrui. Les limites dans lesquelles chacun peut se mouvoir sans nuire à autrui sont marquées par la loi, de même que la limite de deux champs est déterminée par un piquet. Il s'agit de la liberté de l'homme considéré comme monade isolée, repliée sur elle-même.

Le droit de l'homme, la liberté, ne repose pas sur les relations de l'homme avec l'homme mais plutôt sur la séparation de l'homme d'avec l'homme. C'est le droit de cette séparation, le droit de l'individu limité à lui-même ${ }^{44}$. rielles.

Cette séparation est si réelle qu'elle prend des formes maté-

L'application pratique du droit de liberté, c'est le droit de proptiété privée (...)

Le droit de propriété est donc le droit de jouir de sa fortune et d'en disposer «à son gré», sans se soucier des autres hommes, indépendamment de la société; c'est le droit de l'égoïsme. C'est cette liberté individuelle, avec son application, qui forme la base de la société bourgeoise. Elle fait voir à chaque homme, dans un autre homme, non pas la réalisation mais plutôt la limitation de sa liberté.

A son tour, l'égalité

n'est que l'égalité de la liberté définie ci-dessus: tout homme est également considéré comme une telle monade basée sur elle-même ${ }^{45}$.

43. Ibidem, p. 37.

44. Ibidem, pp. 37-38.

45. Ibidem, p. 38 . 
Marx reproche à la bourgeoisie, non seulement d'avoir limité les droits et libertés et donc de les avoir rendus illusoires en tant qu'ils ne se rapportent pas dans leur forme abstraite à la libération de tout l'homme, mais aussi d'avoir «substitué aux nombreuses libertés, si chèrement conquises, l'unique et impitoyable liberté du commerce ${ }^{46}$.

Le fondement et la fin de tels droits n'est donc que « l'homme égoïste, séparé de son semblable et de la communauté », que l'individu «replié sur lui-même, uniquement préoccupé de son intérêt personnel et obéissant à son arbitraire privé », tandis que «la société apparaît comme un cadre extérieur à l'individu, comme une limitation de son indépendance originelle ${ }^{47}$.

Or, cette restriction et cette illusion sont la base du pouvoir politique et de l'Etat que les droits prétendent restreindre. En s'identifiant à l'individu égoïste, l'homme et la société doivent trouver à l'extérieur l'universalité qui doit être imposée comme extériorité et comme contrainte. La fragmentation implicite dans les droits du libéralisme permet paradoxalement l'Etat comme transcendance et universalité, comme pouvoir intégrateur et unificateur.

Mais cette universalité abstraite de l'Etat est aussi une universalité fausse car l'Etat abstrait se particularise comme Etat de classe. Son but prodamé est la «conservation des droits naturels et imprescriptibles de l'homme» (Déclaration de 1791, art. 2), mais par là, concrètement, «la vie politique déclare n'être qu'un simple moyen dont le but est la vie de la société bourgeoise ${ }^{48}$. "L'Etat moderne fait lui-même abstraction de l'homme réel, ou ne satisfait tout l'homme que de façon imaginaire ${ }^{49}$.

Marx rejette donc les droits de l'homme issus de la Révolution française parce que, s'ils font de l'homme une totalité, celle-ci est fermée et abstraite. Ils le coupent téellement de son extériorité, de sorte que celle-ci est elle-même libérée de l'humain

46. MARX, K., Manifeste communiste, Éd. Sociales, p. 17.

47. MARX, K., La question juive, Union générale d'éditions, p. 39.

48. Ibidem, p. 40.

49. MARX, K., Contribution à la critique de la pbilosophie du Droit de Hegel, A. Costes, vol. 1, p. 95 . 
et peut se constituer en totalité autonome. Ils supposent l'opposition et la compétition entre l'intérieur et l'extérieur.

L'extériorité de l'homme, ses produits, la nature, les rapports sociaux, les rapports économiques peuvent se constituer comme pouvoir et domination que permettent et n'arrêtent pas des droits qui ne sont que formels, c'est-à-dire, dont le contenu n'est pas le rapport concret de l'homme et des hommes à leur extériorité. Ce n'est que par le rétablissement de ce rapport dans une totalité qui les comprend positivement que l'homme peut regagner son pouvoir d'émancipation et d'auto-réalisation.

Cette critique des droits et libertés qui débouche sur la critique de l'Etat fonde positivement l'émancipation réelle de l'homme et, par suite, ses droits en tant qu'expression et réalisation de cette émancipation:

Toute émancipation n'est que la réduction, du monde humain, des rapports, à l'homme lui-même. L'émancipation politique, c'est da réduction de l'homme d'une part au membre de la société bourgeoise, à l'individu égoïste et indépendant, et d'autre part au citoyen, à la personne morale.

L'émancipation humaine n'est réalisée que lorsque l'homme a reconnu et organisé ses forces propres comme forces sociales et ne sépare donc plus de lui la force sociale, sous la forme de la force politique ${ }^{50}$.

Le droit et les droits qui expriment cette émancipation et qui ne l'expriment qu'en autant qu'elle est positive, réelle et concrète, sont alors radicalement différents des droits proposés par le libéralisme, car ils ne contiendront pas une abstraction formelle mais la réalité concrète de l'homme avec son extériorité, extériorité non plus autonome, car l'homme s'y prolonge devenant le dynamisme et la source de tout pouvoir conçu comme affirmation de l'humain.

La forme à la fois spécifique et universelle de ce droit est le droit à la différence. En effet, si l'on accepte la description de l'homme réel comme totalité partielle et la liberté comme l'ouverture et rapport d'une totalité partielle à son extériorité, le

50. MARX, K., La question juive, Union générale d'éditions, pp. 44-45. 
concept de différence, concept dialectique, apparaît comme expression adéquate de cette situation. La différence traduit l'état de totalités partielles et ouvertes en voie d'acheminement, en rapport avec d'autres totalités elles-mêmes inachevées. Ce rapport maintient à la fois l'identité ou la continuité et la particularité, la spécificité. L'idée de différence inclut ces deux éléments en évitant la réduction. La différence, c'est la contradiction de la réduction qui réalise l'homologie par la suppression, suppression de la société dans le libéralisme et suppression par suite de l'individu concret, suppression de l'individu dans le collectivisme totalitaire et par suite suppression de la société concrète dans l'abstraction politique.

L'idée de différence doit cependant être précisée. Elle réfère d'abord aux particularités, mais non pas à toute particularité, seulement à celles qui s'établissent dans un rapport de réciprocité conflictuelle qui crée un processus d'identification. C'est en entrant en conflit «que la particularités deviennent des différences, naissent à la différence. Transformées par la lutte, les qualités qui survivent et qui peuvent encore s'affirmer, ne s'affirment plus séparément. Elles ne peuvent se présenter et se représenter que dans leurs rapports réciproques, conflictuels ou apaisés. Ainsi et alors surgit le concept de la différence ${ }^{51}$.

Immédiatement la différence réfère à un contenu concret qui la constitue en tant que telle. Elle ne peut être réduite à une forme abstraite et vide que par convention (au plan logique par exemple).

La différence, parce qu'elle est concrète, résiste "naturellement» à la réduction, à la simplification vulgaire qui nie le spécifique, aux pouvoirs homogénéisants, et donc maintient la complexité et la richesse du sujet. Plus spécifiquement, l'affirmation de la différence bloque tout pouvoir et libère l'essentiel. Par contre, "la réduction apparaît (...) comme l'instrument théorique et pratique, idéologique et stratégique de la recherche du pouvoir. Le projet réducteur se situe sur le trajet de la volonté dominatrice $\gg{ }^{52}$.

51. LEFEBVRE, H. Le manifeste différentialiste, Coll. «Idées», Paris, Gallimard, 1970 , p. 65.

52. Ibidem, pp. 95-96. 
Le droit à la différence signifie donc d'abord le maintien de la spécificité et de l'identité de l'individu, mais dans la croisée des rapports entre la nature, la raison et la société. Le droit à la différence maintient l'extériorité de l'individu mais évite de le réduire, comme totalité, à cette extériorité ou bien à sa particularité ou à sa partialité.

Mais si la différence maintient la complexité du réel concret, elle ne le laisse pas désordonné : elle en fixe le centre, le foyer intérieur et créateur, mais sans que cette centralité dévore la périphérie et l'extériorité, sans quoi elle se détruirait elle-même.

La centralité identifie et spécifie la totalité : elle lui fournit sa forme. Le centre, homme, donne ainsi la forme humaine à l'extériorité de l'homme, à la nature et à la société.

Le centre totalise effectivement ce qu'il influence et rassemble. Il n'a de réalité qu'active, en attirant et repoussant, en incluant dans son aire ou bien en expulsant un contenu, auquel il donne ainsi une forme. En lui-même, le centre n'est rien; et cependant, il fait «tout», puisqu'il fait qu'il y a un tout. La centralité ne peut être que momentanée, relative, provisoire. Elle renvoie à autre chose: les contenus, la périphérie, à l'ailleurs: l'autre centre ${ }^{53}$.

C'est dans et par la différence que l'homme, que l'individu se constitue comme centre et comme sujet, comme sujet de droits en dépassant les particularités mais sans les supprimer, en dépassant son intériorité, sa centralité, mais sans l'abolir, mais plutôt en la réalisant.

Là est le droit à la différence qui «fonde les autres droits concrets et $(. .$.$) en est la source { }^{54}$. C'est sur cette base que le marxisme retrouve ou devrait retrouver les droits de l'homme qui prennent alors un sens et un contenu nouveau et réel. Ils ne jouent plus seulement un rôle «défensif » et abstrait à l'égard du pouvoir mais aussi un rôle positif en ayant pour contenu la réalisation de l'humain dans son intégralité. Ils ont perdu leur partialité et leur «partiellité » qui réduisaient l'homme à l'individu isolé, opposé à son extériorité, à l'individu «privé », coupé de

53. Ibidem, p. 120.

54. Ibidem, p. 45. 
son existence «publique», autre existence et existence comme autre (aliénation). Ces droits sont devenus la condition même de la naissance et de l'affirmation de «l'individu délivré de l'individualisme ${ }^{55}$.

La Déclaration des droits n’est plus rejetée mais complétée. Les droits trouvent un contenu qui en restitue l'intégralité. Ainsi réapparaissent dans ce sens non seulement les droits dans leur formulation ancienne, droit à la liberté, à la liberté de parole, d'association, etc., mais dans de nouvelles formulations, droit au travail, à l'éducation, à la santé, au temps libre, droit à la ville, à la vie urbaine, droit à la modernité, c'est-à-dire droit à l'imaginaire, droit au possible, droit à l'autogestion généralisée, industrielle, urbaine, territoriale ${ }^{56}$, droit à la spécificité individuelle, mais aussi à la spécificité culturelle, morale, nationale, etc.

Les droits de l'homme, de l'homme réel, totalité ouverte, sont aussi restitués, mais sans «l'in-différence» qui les grevait dans la Déclaration libérale et qui en supprimait immédiatement l'universalité concrète pour n'en garder que la forme abstraite.

L'indifférence qui est refus ou impossibilité de l'ouverture permet l'entreprise du pouvoir. C'est dans ce sens que Marcuse, par exemple, a dénoncé avec raison la «tolérance répressive » du libéralisme qui, tout en affirmant les droits de la vérité, se retire indifférente permettant au pouvoir d'imposer «sa» vérité. L'universalité formelle devient particularité réelle, c'est-à-dire pouvoir (la connaissance devient politique !). Et en même temps, la tolérance nie le possible, la nouveauté, comme l'ont démontré les critiques du pluralisme politique ${ }^{57}$. Elle maintient l'ordre du pouvoir et de la répression.

L'on comprend que, dans cette optique, le droit à la différence implique le dépérissement du politique en tant qu'il contient un pouvoir irréductiblement homogénéisant, l'Etat qui

55. Ibidem, p. 168.

56. LEFEBVRE, H., La Révolution urbaine, Coll. «Idées», Paris, Gallimard, 1970 , p. 199.

57. Voir par exemple: H.S. Kariel (ed.), Frontiers of Democratic Theory, Random House, N.Y., 1970, 3 e partie surtout; T.J. Lowi, The End of Liberalism. W.W. Norton and Co., N.Y., 1969; W. Connolly (ed.), The Bias of Pluralism, Atherton Press, N.Y., 1969. 
supprime à son profit et l'individu et la société « pour ne laisser persister que les fonctions économiques ou les structures politiques $\gg{ }^{58}$.

Le droit à la différence implique aussi comme conséquences :

- la fin des séparations, des ségrégations qui créent l'opacité des rapports humains, des rapports sociaux, particulièrement des rapports économiques de propriété privée et de propriété d'Etat (capitalisme privé, capitalisme d'Etat, socialisme d'Etat) ;

- la fin des espaces et des temps préfabriqués qui masquent la polyvalence des possibilités et des façons de vivre;

- la fin du droit contractuel, le droit de la propriété, au profit du droit coutumier;

- la substitution de l'idéal de la croissance pour l'idéal du développement ;

- la fin de l'idéologie et de l'institutionnalisation du fonctionnalisme et du productivisme au profit de la créativité, de l'insolite, de l'imaginaire, etc.

Ce droit à la différence a donc une fonction révolutionnaire et permet de retrouver l'élan révolutionnaire à la base du marxisme car il commande d' «abattre ce système qui formalise et fixe les rapports sociaux, qui les réduit à des formes abstraites». II « reprend et remet en lumière le projet marxiste d'une révolution maximale (totale), projet abandonné au bord de la route ${ }^{59}$.

Or, cette position amène la critique de la pratique actuelle du socialisme, de son impuissance à permettre le droit à la différence, impuissance encore plus grande peut-être que dans la société capitaliste elle-même.

Plus d'un marxiste est conscient de cet échec qui condamne la pratique. Quelques-uns ont apporté des excuses, d'autres des explications, bien peu se sont attardés sur les causes profondes.

58. LEFEBVRE, H., Le manifeste différentialiste, op. cit., p. 158.

59. Ibidem, pp. 155 et 159. 
Le problème demeure entier: pourquoi les sociétés qui se « réclament » du marxisme n'ont-elles pas encore réalisé ce que la doctrine postule? Pourquoi le marxisme qui de toute évidence propose un humanisme dont le point de départ et le point d'arrivée contiennent l'individu, n'a-t-il pas réussi encore, là où on a tenté de le réaliser, à confirmer pratiquement la valeur de la liberté individuelle et des divers droits et libertés, pas plus qu'à établir un système d'égalité concrète? Pourquoi s'est-il développé plutôt dans la plupart des cas en un immense appareil bureaucratique répressif qui étend son emprise non seulement sur la vie politique (il développe et perfectionne l'Etat) et sur la vie économique (le socialisme se transforme en capitalisme d'Etat), mais aussi sur la vie culturelle, esthétique, intellectuelle, etc., sur la vie sociale dans son ensemble?

Les remarques qui suivent et qui servent de conclusion voudraient à la fois esquisser une réponse à ces questions et indiquer le sens d'une recherche.

En effet, à ces questions on peut apporter deux types de réponses, l'un tenant à des facteurs externes à la doctrine et à la pratique du communisme, l'autre interne. La première réponse est la plus facile: elle prend la forme des diverses versions de la thèse stalinienne du «socialisme dans un seul pays» ou de la thèse de «l'étape de transition vers le communisme». Dans l'un ou l'autre cas, il s'agit de faire appel à la conjoncture historique en ignorant les facteurs structuraux.

L'établissement du socialisme en Russie, par exemple, contrairement aux prévisions de Marx, s'est fait dans un pays sousdéveloppé, sortant de la féodalité et non dans un pays développé, tant au plan économique que politique, ayant accompli sa révolution bourgeoise, c'est-à-dire sa modernisation. $\mathrm{La}$ révolution socialiste s'est donc vue confrontée à l'immense tâche de rattraper le retard économique et industriel et pour cela de mobiliser toutes les énergies ${ }^{60}$.

La faiblesse militaire face à l'hostilité des puissances externes créait aussi une «situation de forteresse assiégée», - «situa-

60. Voir par exemple dans Socialist Humanism, les positions de Velyko Korac, p. 11, et de Mihailo Markovic, p. 89. 
tion peu favorable au développement des valeurs humanistes en général et des libertés individuelles en particulier ${ }^{61}$. Faut-it rappeler aussi l'absence de tradition démocratique et humaniste, l'inégal développement social et culturel ?

Mais sans nier l'importance de ces facteurs conjoncturaux qui ont pu accentuer ou renforcer la nature dictatoriale du régime qui s'est établi sous le couvert du socialisme et qui ont pu faire «obstacle à l'intégration par ces sociétés de valeurs humanistes et libérales », ne pouvons-nous pas, ne devons-nous pas nous interroger plutôt sur un ou des facteurs structuraux qui auraient bloqué (rendu impossible) l'affirmation intrinsèque des droits et libertés implicites à la doctrine? Dans ce cas, toute société avec la même structure développerait la même propension à la bureaucratie et à la dictature.

Lucien Goldmann, en Occident ${ }^{62}$, fut peut-être le premier à répondre affirmativement et à tenter d'identifier ces causes structurelles, quoique encore confusément. Il faut le citer ici :

C'est en effet la pensée marxiste qui a mis en lumière la relation historique entre l'existence de la production pour le marché et les valeurs libérales et individualistes de l'humanisme bourgeois, la conjonction, propre à cette structure historique particulière, du progrès de la maîtrise par l'homme de la nature et de la société, de la liberty to (qui caractérise l'ensemble de l'histoire), avec un développement important et notable des libertés individuelles et de l'humanisme individualiste, de la liberty from. Il était donc naturel et prévisible - bien que ni Marx et Engels ni les penseurs marxistes ultérieurs n'y eussent jamais pensé - que la suppression de la production pour le marché et son remplacement par une planification centralisée dans les sociétés socialistes changeât sur un point précis la direction de l'évolution, en favorisant un très grand développement du conformisme de l'intégration des individus au groupe et de l'acceptation des normes et opinions reconnues et approuvées par ce dernier ${ }^{63}$.

61. GOLDMANN, L. op. cit., p. 308.

62. Il semble que déjà certains économistes soviétiques, dès les années 1920 , V.A. Bazarov, V.G. Gorman et I.T. Smilga, en aient eu le pressentiment.

63. GOLDMANN, L., op. cit., p. 309. 
Et Goldmann de tenir pour fondamentale la leçon de l'expérience yougoslave qui a permis,

la découverte du fait que la socialisation des moyens de production n'implique pas nécessairement, comme le pensaient Marx et les marxistes ultérieurs, une planification centralisée intégrale et la suppression du marché.

La grande conquête de la démocratie socialiste yougoslave, l'autogestion ouvrière, est du point de vue théorique non seulement le moyen d'assurer une démocratie effective, mais aussi l'union d'une socialisation très poussée de la propriété des moyens de production, socialisation qui permet de supprimer l'exploitation de l'homme par l'homme et, en tout cas, une partie notable des manifestations de la réification, et du maintien d'une production pour le marché, susceptible de constituer le fondement d'un développement réel et authentique de la liberty from, des valeurs humanistes de liberté en général, de liberté d'expression en particulier et de dignité individuelle. ${ }^{64}$.

Les indications de Goldmann m'apparaissent d'une extrême importance: la négation des mécanismes du marché oblige à développer un appareil de régulation artificielle qui ne peut fonctionner sans impliquer tous les secteurs de la vie sociale, car ceuxci sont liés les uns aux autres. Le principe de Saint-Simon, repris par Engels, «du gouvernement des hommes à l'administration des choses » apparaît exclusivement une vue de l'esprit car, entre les hommes et les choses, il y a continuité : ils sont également inclus dans une totalité.

On connaît la condamnation marxiste de l'économie de marché. Pour Marx, le marché commande l'aliénation du travailleur en déshumanisant le produit de son travail et son travail lui-même dans le fétichisme de la marchandise dont la forme la plus abstraite est la monnaie. Le marché transforme les relations sociales et humaines en relations abstraites qui prennent forme dans la division du travail qui rend abstrait le travail lui-même en le privant de la créativité et en transformant son produit en produit abstrait où l'humain ne se retrouve plus. Le raisonnement de Marx confond ici, semble-t-il, la division sociale du travail et la division du travail dans l'entreprise.

64. Ibidem, p. 310. 
Mais aussi, comme la plupart des socialistes qui l'ont précédé, Marx condamne le marché comme mécanisme de régulation économique parce que la loi de l'offre et de la demande (avec les phénomènes qui en dépendent: la surproduction et la crise) ne peut se concilier avec une véritable rationalité et efficacité économiques. Elle est la source de gaspillage de biens matériels et d'énergie humaine.

Ces raisons théoriques en rencontrant des éléments conjoncturaux de nature sociologique et politique ont évidemment amené le socialisme scientifique à rejeter les mécanismes de marché au profit d'une planification centralisée, exclusive de tout autre mécanisme de contrôle et d'adaptation. Ce type de planification eut rapidement des effets bénéfiques en fonction des buts conjoncturaux : elle permit de réquisitionner les ressources et d'établir un taux unique d'accumulation en même temps qu'un taux extrêmement bas de consommation au profit de l'industrialisation et du développement militaire.

Mais se développa, sur la base de ces structures et mécanismes économiques et dans le cadre d'un appareil politique d'essence militaire (le parti), une élite économico-politique dont l'intérêt et l'expression se trouvaient dans le pouvoir. Tout partage ou réduction de celui-ci ne pouvait que la menacer.

Ainsi il est possible de conclure que si l'établissement de la dictature fut favorisé par la conjoncture historique, elle repose ultimement sur un élément structurel qui peut être décrit ainsi: toute économie complexe a besoin d'un principe de régulation, soit automatique (le marché), soit artificiel (le contrôle central direct); l'élimination du marché comme régulateur amène un modèle économique dont le principe de régulation tient à un contrôle central qui gère directement l'ensemble de l'économie comme une entreprise unique fixant les buts, les moyens et les règles de toute activité économique. Or cette structure entre en contradiction avec la structure concrète de la société complexe d'intérêts et de pouvoirs opposés et inégalement développés. Pour réaliser sa finalité, elle doit non seulement se développer dans un pouvoir économique centralisé assurant la planification et la régulation de l'économie, mais aussi dans un pouvoir politique homogénéisant les distinctions et les différences. Une société 
privée de la régulation du marché ne peut être démocratique sans que ceci ne signifie qu'une société de marché l'est nécessairement. Dans tous les sens, la démocratie économique est toujours la condition de la démocratie politique.

La démocratisation du socialisme exige la fin de la planification centralisée dans sa forme actuelle et comme mécanisme unique de régulation. Elle exige aussi le retour à une forme d'économie de marché (qui n'a d'ailleurs jamais complètement cessé dans ses formes déviantes - le marché noir). Ce n'est qu'à cette condition que le droit à la différence sera la loi théorique du développement de l'homme et de l'individu, la loi théorique et pratique $\mathrm{d}^{\text {' }}$ une association où le libre développement de chacun est la condition du libre développement de tous ».

Mais la restauration du marché n'implique pas le retour éhonté au capitalisme. Le marché est polyforme; il a existé avant le capitalisme dans des formes diverses liées à des modes de production divers. Il peut exister sous une forme socialiste où le circuit de la marchandise ne sera pas celui du capitalisme (A-M-A) où la monnaie est principe et fin du mouvement, et l'accumulation le résultat, mais celui où la marchandise comme valeur d'usage est au début et à la fin (M-A-M), où la monnaie est réduite à un pur intermédiaire dans le mécanisme d'échange et où le support du mouvement est la relation besoin (ou désir) satisfaction (ou jouissance).

Ainsi conçu, le marché peut se concilier avec une planification qui reçoit surtout ses informations (information sur l'offre et surtout la demande) du marché tout en agissant sur le marché lui-même. Quand le marché et la planification sont conciliés avec des formes diverses d'appropriation et de gestion (possession publique et autogestion des producteurs; coopérative; gestion sociale, etc.), les conditions pour une structure sociale, politique et économique qui réponde à la base fournie par la conception marxiste de l'homme comme totalité partielle et ouverte et la conception du droit comme essentiellement droì à la différence sont réunies.

Ceci est sommaire, beaucoup de problèmes ont été laissés sur le bord du chemin en cours de route, beaucoup de questions 
demeurent en suspens, mais il y a là une direction qui émerge dans certains pays communistes, qu'il faudra observer. Car c'est peut-être de là que viendra un nouveau socialisme pour l'homme libéré.

Université d'Ottawa 